\title{
BMJ Open Improving chronic pain management with eHealth and mHealth: study protocol for a randomised controlled trial
}

\author{
Irene Jaén (10 , , Carlos Suso-Ribera, ${ }^{1}$ Diana Castilla, ${ }^{2,3}$ Irene Zaragoza, ${ }^{3}$ \\ Azucena García-Palacios, ${ }^{1,3}$ Jose Luis Gómez Palones ${ }^{4}$
}

To cite: Jaén I, Suso-Ribera C, Castilla D, et al. Improving chronic pain management with eHealth and mHealth: study protocol for a randomised controlled trial. BMJ Open 2019;9:e033586. doi:10.1136/ bmjopen-2019-033586

- Prepublication history and additional material for this paper are available online. To view these files, please visit the journal online (http://dx.doi org/10.1136/bmjopen-2019033586).

Received 12 August 2019 Revised 12 November 2019 Accepted 14 November 2019

Check for updates

(C) Author(s) (or their employer(s)) 2019. Re-use permitted under CC BY-NC. No commercial re-use. See rights and permissions. Published by BMJ.

${ }^{1}$ Basic Psychology, Clinical Psychology and Psychobiology, Universitat Jaume I, Castello de la Plana, Spain

${ }^{2}$ Psychology and Sociology, Universidad de Zaragoza,

Zaragoza, Spain

${ }^{3}$ CIBER of Physiopathology of Obesity and Nutrition CIBERobn, CB06/03, Instituto de Salud

Carlos III, Madrid, Spain

${ }^{4}$ Pain Clinic, Hospital General de Castellon, Castellon de la Plana, Spain

Correspondence to

Irene Jaén; ijaen@uji.es

\section{ABSTRACT}

Introduction Chronic pain has become a matter of public health concern due to its high prevalence and because public costs associated with treatment and disability increase each year. Research suggests that limitations in the traditional assessment of chronic pain patients limit the effectiveness of current medical treatments. The use of technology might serve change patient traditional monitoring into ecological momentary assessments, which might be visualised by physicians live. This study describes a randomised control trial designed to test the utility of a technology-based solution for pain telemonitoring consisting of a smartphone app for patients and a web application for physicians. The goal of this study will be to explore whether this combination of eHealth and mHealth improves the effectiveness of existing pain treatments.

Methods and analysis Participants will be 250 patients randomly assigned to one of these two conditions: treatment-as-usual (TAU) and TAU +app+ web. All participants will receive the usual treatment for their pain. Only the TAU +app+ web group use Pain Monitor app, which generates alarms that are sent to the physicians in the face of previously established undesired events. Physicians will be able to monitor app reports using a web application, which might result in an adjustment of treatment. We anticipate that the use of Pain Monitor plus the therapist web will result in a reduction of pain intensity and side effects of the medication. Improvements on secondary outcomes, namely fatigue, mood, pain interference, rescue medication use and quality of life, are also expected. Mixed repeated-measure multivariate analyses of variances will be conducted to investigate whether there are differences between preassessment and postassessment scores as a function of the experimental condition.

Ethics and dissemination Ethical approval from the Hospital General Universitari de Castellon was obtained. The findings will be published in peer-reviewed journals. Trial registration number NCT03606265

\section{INTRODUCTION}

Pain can be defined as 'an unpleasant sensory and emotional experience associated with actual or potential tissue damage, or described in terms of such damage ${ }^{1}$ and can
Strengths and limitations of this study

- In the present randomised, controlled clinical, an integrative e-health and $\mathrm{m}$-health solution for chronic pain management is implemented.

- Patient monitoring is performed remotely in an ecological and momentary manner with a smartphone app.

- Patient responses to the app might generate alarms in the presence of unwanted clinical events.

- Physicians can track patient evolution at any time on a website and receive clinical alarms daily.

- Study limitations include the fact that physicians are not blinded to the patients' condition and the rigidity of the app assessment protocol.

only be understood as an interplay between 'sensory, emotional, cognitive and social components'. ${ }^{2}$ Although pain often is acute and disappears as tissues heal, sometimes pain persists for long periods of time and becomes chronic. For instance, it has been reported that $15 \%$ of individuals admitted to trauma hospitals due to a severe injury and 15\%-60\% of patients after surgery will continue to experience chronic pain months and years later. ${ }^{3}$ In general, a cut-off of 3-6 months is used to define the transition from acute/subacute to chronic pain. ${ }^{4}$

The aforementioned chronification of pain is becoming a major public health problem across the globe. ${ }^{5}$ We refer here to primary chronic pain, a pain associated with important interference on functioning and/or emotional distress, which cannot be better accounted for by any other condition. ${ }^{6}$ Specifically, epidemiological studies indicate that the prevalence of this disease in the adult population ranges from $19 \%$ to $38 \%$ worldwide. ${ }^{7-10}$ Furthermore, the increase in life expectancy and the ageing of the population is likely to have an important impact on the 
number of individuals experiencing chronic pain, since the prevalence of this syndrome boosts dramatically with age. ${ }^{11}$ For instance, it is expected that the population of chronic pain individuals will be doubled in 2050 for people older than 65 years and tripled for people over 80 years of age.$^{12}$ Thus, chronic pain is a major public health challenge due to its high prevalence in the population and high direct and indirect costs for the institutions and the individuals. ${ }^{1314}$

Indeed, chronic primary pain (eg, fibromyalgia or nonspecific low back or neck pain, to name some examples) is imposing a huge burden in our societies as this disease has become one of the leading causes of years lived with disability globally. ${ }^{1516}$ Not surprisingly, as a result of the growing concern about this disease, there have been numerous attempts to improve treatments for pain in the past decades. However, recent reviews on the effectiveness of numerous interventions, including medical treatments, psychological therapy, physical rehabilitation or a combination of these, indicate that the effectiveness of existing treatments is, on average, only modest. ${ }^{17-19}$ While there might be numerous factors explaining the limited effectiveness of current interventions for pain, including unexplored biomechanical mechanisms or genetic factors, patient characteristics or therapists' training, some authors have pointed to methodological shortcomings as key elements explaining the modest effectiveness of pain interventions. Specifically, the way assessment is currently performed (ie, a single measure of pain intensity performed episodically during the onsite appointments) has been argued to impact negatively in the ability of existing interventions to achieve more reliable and powerful changes in patient outcomes. ${ }^{20}{ }^{21}$ For instance, a single rate of pain intensity has been shown to be an unreliable measure of pain as this experience can vary dramatically within the same day and across days. ${ }^{22-24}$ In addition, pain is frequently assessed retrospectively, which is known to lead to recall bias and to decrease the accuracy of pain ratings ${ }^{25}$ and does not allow for timely responses to undesired events, so these often take place time after the problem occurred. ${ }^{21}$

As a consequence of the above, ecological momentary assessment (EMA), which refers to the assessment of pain repeatedly and in real life, has received renewed interest in the past years in the pain literature and is now considered by many as the gold standard method to assess the pain experience. ${ }^{26-29}$ Traditionally, EMA has been difficult due to the limitations and costs of repeated measurement procedures (ie, paper diaries or phone calls). However, with the explosion and availability of smartphones, EMA has become easier than ever and immediate communication between the patient and the physician is now a more feasible practice. ${ }^{30}$

It has been argued that this change in the assessment paradigm towards ecological daily telemonitoring using apps will improve treatment effectiveness and reduce costs if used to respond to patient reports quickly. ${ }^{21}{ }^{31}$ Indeed, there is evidence to suggest that smartphones are useful tools to be used for the assessment of pain core outcome measures in chronic pain settings. ${ }^{21} 3233$ However, the extent to which this EMA of pain patients can effectively lead to better practices in pain medicine is still unknown. For this purpose, we developed a technology-based solution that integrated a pain and symptom tracking app for patients and a web for physicians where app-generated alarms are received daily and patient app responses can be monitored in real time.

With the previous goal in mind, in the present parallel group, superiority trial we will use the Pain Monitor app (https:// play.google.com/store/apps/details?id=painmonitor.srccode), which was developed by a team of psychologists and an engineer with the collaboration of physicians and nurses and has been recently validated in clinical settings, ${ }^{21}$ together with a web for the physicians where app responses and alarms can be tracked in real time to facilitate the professional's decision-making process. As we will explain in more detail in the Methods section, Pain Monitor assesses a number of pain-related outcomes (ie, pain intensity, pain interference, anxiety and depression and use of pain-related health resources) and the most frequent side effects of medical treatments for pain. In the study, patients will be randomly assigned to a treatment-as-usual condition (TAU) or to a TAU with the support of the patients' app and the physician's web. We anticipate that the use of the web application linked with the smartphone app (TAU +app+web condition) will improve the effectiveness of usual treatments resulting in reduced pain intensity and less frequent side effects of the medication after 1 month of medical treatment. Additionally, we expect that this group of patients will present additional improvements on secondary outcomes, including mood (depression and anxiety), pain interference, pain catastrophising and use of pain-related health resources in the past month as secondary gains of reducing pain levels, as suggested in the literature. ${ }^{34}$ We also expect that the rapid detection of treatment undesired events will rapidly minimise threats to the patient's quality of life and mood.

\section{METHOD}

\section{Study design}

The current investigation is a randomised superiority clinical trial composed of two parallel groups (1:1 allocation ratio): (1) TAU and (2) TAU +app+ web. In the study, participants in the TAU condition receive the usual pain treatment by the physicians working at the pain unit (ie, pharmacological treatment or infiltration). Participants included in TAU +app+ web group receive the usual treatment for their pain plus daily monitoring of their symptoms and pain experience with the Pain Monitor app during 1 month. In the TAU +app+web condition, alarms are generated in the presence of previously established undesired events, which have been previously determined by the physicians at the pain clinic (eg, pain intensity is higher than 7 in an 11-point numerical scale during three 


\begin{tabular}{rccc}
\hline & \multicolumn{3}{c}{ STUDY PERIOD } \\
\hline & $\begin{array}{c}\text { Pre- } \\
\text { intervention }\end{array}$ & Intervention period & Close-out \\
\hline TIMEPOINT & 0 & $T$, & $T_{2}$ \\
Pre-Intervention & Between assessments & One month follow-up \\
\hline ENROLMENT: & & \\
\hline Eligibility screen & $\times$ & \\
\hline Informed consent & $\times$ & \\
\hline Allocation & $\times$ & \\
\hline
\end{tabular}

\begin{tabular}{rlll}
\hline INTERVENTIONS: & & \\
\hline Medical treatment & App condition only & \\
\hline App use & & & \\
\hline ASSESSMENTS: & & & \\
\hline Demographics & $\times$ & App condition only & $\times$ \\
\hline Primary outcomes & & App condition only & $\times$ \\
\hline Pain intensity & $\times$ & & \\
\hline Physical symptoms & $\times$ & App condition only & $\times$ \\
\hline Secondary outcomes & & App condition only & $\times$ \\
\hline Pain interference & $\times$ & App condition only & $\times$ \\
\hline Mood & $\times$ & App condition only & $\times$ \\
\hline Fatigue & $\times$ & App condition only & $\times$ \\
\hline Rescue medication & $\times$ & $\times$ & \\
\hline Quality of life & & & \\
\hline
\end{tabular}

Figure 1 Study schedule of enrolment, interventions and assessments.

consecutive days). Physicians are able to monitor these patients' app reports using a web application created for this purpose (https://monitordolor.dolortic.com/). Thus, phone calls can be conducted in the presence of alarms in order to change or discontinue the medical treatment when necessary. If the study results indicate that the use of technology leads to better outcomes, participants in the TAU condition will be informed about these findings and will be offered the possibility to use the app after study participation. In the TAU condition only, assessment is performed as usual, that is, using self-report

\section{Box 1 Inclusion criteria}

The patient is over 18 years of age.

- The patient has a mobile phone with Android operating system (the app is currently only available for Android, which is the operating system used by more than $80 \%$ of users in Spain). ${ }^{49}$

- The patient has the physical ability to use the application.

A new treatment plan is started during the first week after study onset.

- The patient does not present psychological and/or cognitive alterations or problems with language that make his/her participation difficult.

- The patient voluntarily wants to participate and signs the informed consent form. measures administered onsite at the beginning and the end of the study (1 month later).

Neither the physicians nor the patients will be blind to the treatment condition assigned.

Physicians will not be blind because they will receive alarms from the TAU +app+web participants only. Patients will not be blind because only those in the TAU +app+web condition will be using technology in addition to usual treatment and because patients in the TAU condition must know that there is no telemonitoring in their condition.

All items from WHO Trial Registration Data Set are showed in the online supplementary file 1 . The recruitment started at the end of the same month. Standard Protocol Items: Recommendations for Interventional Trials guidelines were followed to design the trial. The participant timeline (ie, schedule of enrolment, interventions and assessments) is shown in figure 1. Recruitment is currently ongoing and is expected to end in November 2019.

\section{Sample}

Participants will be 250 consecutive chronic pain patients attending the pain clinic at the Hospital General Universitari de Castello (Spain) for the first time. Required sample size was calculated using $G$ *Power. ${ }^{35}$ Although the a priori calculation resulted in 198 participants, the sample size was increased to 250 considering a drop-out rate of $27 \%-30 \%$ based on previous studies. ${ }^{3637}$ Thus, 125 participants were assigned to each condition. Randomisation of participants was performed by an independent researcher using a computer-generated sequence with Randomizer. ${ }^{38}$ Inclusion criteria are shown in box 1 . Only patients for whom a change in the treatment is planned (eg, an epidural infiltration or a change in the prescribed medication) will be included in the study (this includes both new and consecutive patients). The reason for doing this is that the utility of the technology is expected to be maximised during the onset of new treatments, as opposed to those cases in which the treatment plan is already well established.

In the study, all participants are identified using an alphanumeric code. In the case of participants in the TAU +app+web condition, this code is automatically generated by the app. Thus, the database generated by the app is anonymised and the app only collects the international mobile equipment identity. The association between app codes and patient identifiable characteristics is stored locally at the pain clinic. All data storage procedures follow the European law and data protection rules (European Union General Data Protection Regulation 2016/679 of the European Parliament and of the Council of 27 April 2016). Important protocol modifications will be notified and require the approval of the Ethics Committee of the Hospital General Universitari de Castello. Approved changes will be made public at ClinicalTrials.gov. All the participants read and sign an informed consent form before randomisation (see online 
supplementary file 2). Patients, who do not agree with the assigned condition, are given the opportunity to be allocated to the preferred condition, but are not used in the analyses. Any changes to modify the assigned condition are accepted at any time during the study, again resulting in an exclusion from the study. Changes in the medication or improvement of disease do not result in study discontinuation. Disease worsening is not expected to be associated with the inclusion of the app but, if existent, will result in the discontinuation of app use.

\section{Procedure}

The study is conducted at the pain clinic of the Hospital General Universitari de Castelló. The study is advertised by the physicians to all consecutive patients attending the pain clinic for the first time. To ensure enrolment, physicians will emphasise the importance of active patient participation in research in general and in selfmonitoring in particular. Patients interested in participating are directed to another office where the lead author, IJ, explains the study procedures in more detail and ensures their eligibility. $\mathrm{IJ}$ is in charge of increasing adherence to the treatment (ie, app) by explaining the utility of the study and by contacting patients when an alarm informing of low app adherence (ie, more than three consecutive days without response) is received. All participants are provided with an information sheet and sign the informed consent. After participation acceptance, participants are assigned to one of the experimental conditions (TAU or TAU +app+web), which had been previously randomised by an external researcher. All participants then complete a paper-and-pencil assessment protocol in order to control for differences between the two assessment formats (app vs pen and pencil) and to compare both conditions using the same assessment approach. In addition to this paper-and-pencil evaluation, patients in the TAU +app+web condition download and instal the Pain Monitor app into their phones. Once they instal the app, they answer to an initial assessment and then complete two measures daily (10:00 and 19:00 hours) during 1-month (study duration). Finally, an end of study appointment is set (1 month later) to conduct the postassessment evaluation. Due to difficulties in transportation or availability, the postassessment intervention can either be completed onsite or via an on-line survey.

\section{Pain monitor}

The Pain Monitor app (figure 2) has been developed by a group of pain psychologists and an engineer, with the collaboration of physicians and nurses specialised in pain care. Pain Monitor is composed of several pain-related items which are to be answered twice a day at preset times (10:00 am and 19:00 hours, with a 2-hour flexibility) during 30 days. The app content has been previously validated with chronic pain patients at the pain unit of the Vall d'Hebron Hospital. ${ }^{21}$ This assessment protocol contains sociodemographic items (ie, age, sex and education level, among others) which are evaluated on the first day of app use only, as well as a number of pain-related outcomes that are evaluated daily, which have been selected following recent guidelines on core outcome domains for pain treatments. ${ }^{39}{ }^{40}$ Constructs in the app, including pain intensity, pain interference, anxiety, depression, catastrophising, social support, acceptance
A

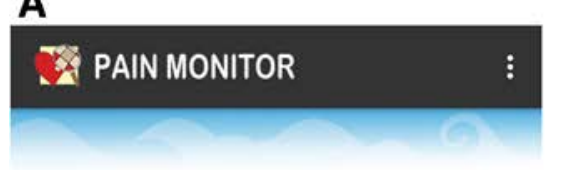

\section{INSTRUCTIONS}

1. Answer to some questions on the characteristics of your problem

2. During 30 days, answer to a set of questions twice a day (morning and evening). The app will inform you automatically when to respond (default times are 10 a.m. and 7 p.m. but you can change that after the first set of questions and anytime while using the app by clicking the "Configuration" menu on the top right of the app)

3. VERY IMPORTANT. Whenever you have an acute pain episode, open the app and answer to a few questions.

4. Contact us at dolorcronico@uji.es if you need assistance
B

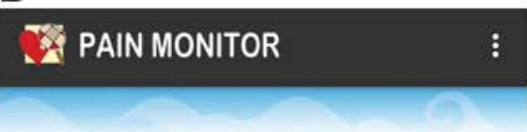

Please indicate the intensity of your CURRENT PAIN:

$0=$ No pain

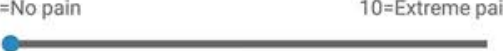

-
C

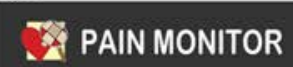

:

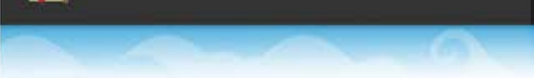

Please indicate the intensity of your CURRENT FATIGUE:

Selected score: 5

$0=$ No fatigue

$10=$ Extreme fatigue 
and coping, among others, are measured with a single item to reduce the burden of daily assessment, each of which was adapted and validated against well-established paper-and-pencil measures. ${ }^{21}$ Additionally, the assessment protocol includes a list of side effects created ad hoc based on the literature findings on the most frequent adverse effects of pain treatments, ${ }^{414}$ as well as measures of treatment adherence, use of rescue medication, neuropathic characteristics of pain and use of medical services in the past month. All app items can be found in online supplementary file 3 .

The app generates alarms in the presence of predefined events (see online supplementary file 4 for the alarms set in the present study in collaboration with the participating physicians). These alarms are sent to the physicians early in the morning on working days so that they can decide whether an action from their side is required (eg, calling the patient and setting an earlier appointment or suggesting a change in the medication). For this study, a website linked to the app was created for the physicians to observe patient alarms and evolution live. Examples of the physician web are presented in figure 3. Physicians are only asked to check the website when an alarm happens, but they are allowed to check any patient status at any time.

\section{Interventions}

Five physicians at the pain clinic of the Hospital General Universitari de Castelló participate in this study. All patients in the study receive the usual treatment for their pain irrespective of their assigned condition. However, a change in treatment might occur in the TAU +app+web condition at the discretion of the physicians in charge of treatment after receiving an alarm and consulting the web page with the graphical representation of patient app responses. As usual, patients in the TAU condition without the app are not contacted by the physicians between appointments. It is important to note that both patients in the TAU only and patients in the TAU +app+web condition are allowed to attend to the emergency services or the family physician in the event of an emergency at any stage of the study due to ethical reasons. At the end of the study, this practice is investigated for each participant in the final assessment.

\section{Assessment plan}

All participants in the study fill in a number of questionnaires in a paper-and-pencil format at the beginning and at the end of the study. This assessment protocol includes sociodemographic information, sickness work absence during the past month, use of pain-related health resources in the past month (ie, emergency services, family physician or pain clinic), pain-related physical symptoms experienced in the past week (ie, side medication effects), the Brief Pain Inventory (pain severity and interference) ${ }^{43}$ the Pain Catastrophising Scale ${ }^{44}$ and the Hospital Anxiety and Depression Scale. ${ }^{45}$ In addition to this paper-and-pencil evaluation, participants in the TAU +app+ web condition also instal the Pain Monitor app and complete a preintervention assessment in the app after the paper-and-pencil evaluation. Both baseline assessments include the same content and are duplicated to provide further evidence for the validity of app content. After this pretreatment evaluation, participants in the TAU +app+web group are asked to answer to the app assessments twice a day during 1-month (study duration). A push-up system notifies the patient about the need to respond to the app evaluation at 10:00 and 19:00hours. These times can be adjusted by the patient with a 2-hour flexibility from the preset times.

Daily morning and evening assessments differ in a number of items. Some items are asked twice a day (ie, pain intensity, sadness, anxiety), while others are only administered in the morning (eg, interference of pain on sleep) or in the evening (eg, activity level during the day, interference of pain on daily activities, or physical symptoms experienced during the day).

Finally, 30 days after the treatment onset (ie, first evaluation), both groups complete a postassessment protocol. The measures included in this final evaluation are similar to the ones included in the baseline assessment, with the inclusion of a measure of negative events experienced during the study period and the evaluation of perceived change due to treatment.

In the study, primary outcomes are pain intensity and the number of side effects of the medication reported in the app, while secondary outcomes include mood (depression and anxiety), pain interference, pain catastrophising and use of pain-related health resources in the past month.

Note that app reports in the TAU +app+web condition are not used to determine treatment effectiveness compared with the TAU only condition because in the latter condition participants do not use the app. Therefore, app responses are only used for telemonitoring and early detection of treatment problems that result in an alarm to the physicians. The comparison of both conditions will be made using the traditional paper-and-pencil evaluations, which will be available for both groups. Additionally, the number of alarms and the physician's responses to such alarms (eg, change in treatment strategies) will be registered. This information will be used to get better insight into the utility of the integrated technology to improve treatment efficacy.

\section{Patient and public involvement}

In the current study, patients or the public will not be involved in the design, or conduct, or dissemination of the research.

\section{Data analysis}

The aim of the present study is to explore the effect of an integrated technology-based solution for chronic pain monitoring (an app that monitors pain patients daily and sends clinical alarms to physicians and a web for physicians that graphically represents patient evolution as reported 


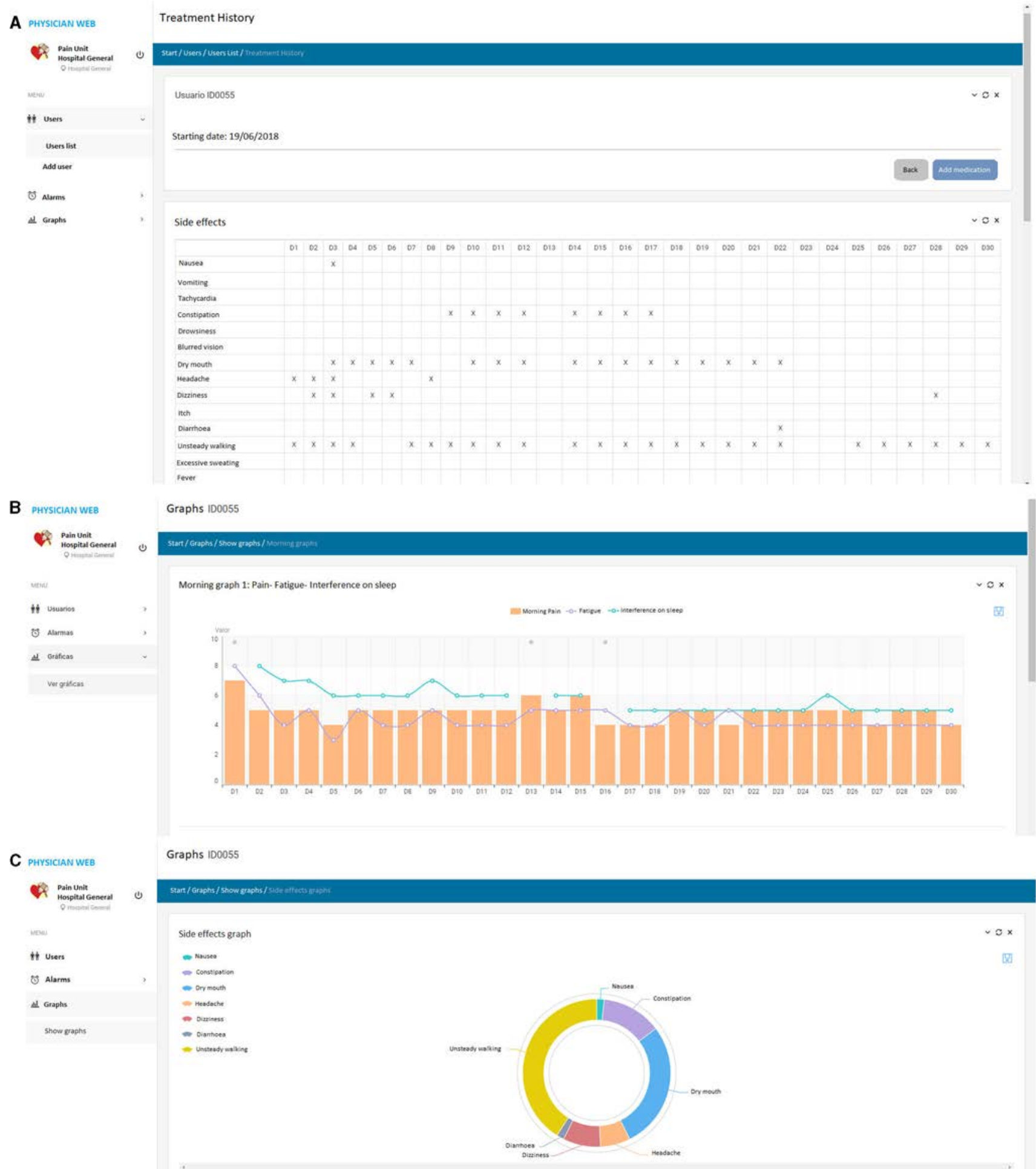

Figure 3 Examples of the web for the physician. (A) Patient's side effects during 30 days. (B) Patient morning values on pain, fatigue and Interference on sleep. (C) Distribution of patient side effects.

in the app) compared with the usual treatment where monitoring is made using a paper-and-pencil, episodic, onsite evaluation. With this aim in mind and completer analyses will be performed following the recommendations of the Consolidated Standards of Reporting Trials guidelines (http://www.consort-statement.org/). First, the two conditions will be compared at baseline in the different continuous measures with a between-group analysis via a t-test to ensure that randomisation indeed resulted in comparable groups prior to intervention. $\mathrm{X}^{2}$ tests will be used for all the categorical variables. To evaluate our hypothesis, mixed repeated-measure multivariate analyses of variances (MANOVAs) will be conducted to investigate whether there are differences between preassessment and postassessment scores as a function of the experimental condition (TAU or TAU +app+web). 
Distribution normality and homoscedasticity assumptions will be tested by means of Kolmogorov-Smirnov and and Levene tests, respectively, and a Mann-Whitney U test and Brown-Forsythe F-test will be used where necessary. Effect size will be calculated to complement the MANOVA results with the standardised mean difference (Cohen's d) for both between and within group analyses. This is a novel study and effect sizes are difficult to anticipate. However, we expect to find larger (ie, moderate) between-groups effect sizes for primary outcomes (ie, pain intensity and number of side effects of the medication) when compared with secondary outcomes since medical interventions do not specifically focus on these symptoms (ie, pain interference, mood, fatigue, rescue meditation use and quality of life). The analysis will be performed by CS-R, who will be blinded to the treatment allocation. Only the present study authors will have access to the final trial dataset.

Regarding dropouts, we will choose a strict criterion and the analyses will only include participants who complete both the pre and the postassessments. Because of the short duration of the trial (1 month per patient) and the minimal risks expected from the use of the app, a data monitoring committee will not be required. Despite the previous, an alarm has been set so that the physicians are warned if a patient fails to respond to the App during three consecutive days (ie, an indirect measure of potential dropouts attributable to the App use). If this happens, the physicians will call the patient and explore the reasons for discontinuation and try to obtain a posttreatment assessment to reduce bias.

\section{DISCUSSION}

Pain assessment is a complex process characterised by a high variability between and within days, which is usually performed by clinicians using self-report, onsite, single ratings which are based on recall. ${ }^{46} 47$ EMA using smartphone apps appears to be an innovative and promising alternative to these traditional assessment methods ${ }^{48}$ as smartphone apps have demonstrated to be accurate tools to assess pain intensity and related variables from the patients' home, thus facilitating telemonitoring and contributing to the personalisation of medical interventions by rapidly adjusting treatments to every individual as a result of telemonitoring. ${ }^{25}$

In the present study protocol, we describe a randomised controlled trial designed to test an integrative technologybased solution for chronic pain monitoring consisting of a web application for the healthcare professional, which is linked to the patient's app (ie, Pain Monitor). Specifically, we want to explore whether the use of this integrative technology improves the effectiveness of the usual treatment for this population thanks to telemonitoring and the rapid detection of unwanted events. We expect that the use of Pain monitor, with the support of therapist's web, will result in reduced pain intensity and less frequent side effects of the medication after 1 month of medical treatment due to the professional's rapid reaction in the presence of undesired outcomes. Note that the study goal is not the explore the feasibility of implementing the use of the integrative technology for patient long-term use, but to explore its utility and acceptability when used in the short term (eg, during a month) in a critical treatment stage (ie, after the onset of a new treatment plan, when pain is not well controlled and treatment tolerance is unclear).

To our knowledge, this is the first study to assess the effectiveness of this type of integrative technology solution (ie, a therapist web site linked to a patient smartphone app) for the telemonitoring of patient symptomatology in chronic pain. If our hypothesis is confirmed, our findings will serve to demonstrate the feasibility and utility of smartphones and specialised webs for therapists so that they can be implemented in specialised care contexts (ie, pain clinics). Likewise, our results will provide important information about the potential benefits of smartphone apps for the personalisation of pain treatments (ie, treatment can be rapidly personalised to a given patient as a function of individual responses reported in the app). Ultimately, this might help change the model of care for this chronic disease (ie, episodic, onsite assessment and treatment), since the use of this integrative technology system allows for a continuous and remote evaluation and intervention, providing a faster response to the patient needs and improving self-management and empowerment of patients who attend pain clinics as they become important agents of treatment effectiveness by being in charge of daily reporting of pain-related experiences in the app. In sum, the results of the present investigation could serve an important first step towards the implementation of apps and other Information and Communication Technologies in health services.

\section{Twitter Irene Jaén @irenejaenpsic}

Contributors All authors were strongly involved in the study conceptualisation and design and have reviewed and discussed the manuscript. IJ and CS-R prepared the first draft of the manuscript, which was then reviewed by AG-P, DC, IZ and JLGP. After changes were incorporated, a final version was approved by all authors. IJ and JLGP are currently in charge of recruitment and IJ and CS-R will be in charge of data analysis.

Funding Funded by Plan de Promoción de la investigación Universitat Jaume I. Ref UJI-B2016-39 and a Predoctoral Grant (PRED0C/2017/26) by the Universitat Jaume I to IJ. The first grant allowed for the development of the technological systems used in the study (physician website and link to the app). The second grant serves to pay the salary of the lead researcher and predoctoral candidate, IJ.

Competing interests The intellectual property of the Pain Monitor app is owned by coauthors CS-R, DC, IZ and AG-P.

Patient consent for publication Obtained.

Ethics approval Ethical approval from the Hospital General Universitari de Castelló was obtained, in accordance with the Declaration of Helsinki.

Provenance and peer review Not commissioned; externally peer reviewed.

Open access This is an open access article distributed in accordance with the Creative Commons Attribution Non Commercial (CC BY-NC 4.0) license, which permits others to distribute, remix, adapt, build upon this work non-commercially, and license their derivative works on different terms, provided the original work is properly cited, appropriate credit is given, any changes made indicated, and the use is non-commercial. See: http://creativecommons.org/licenses/by-nc/4.0/. 
ORCID iD

Irene Jaén http://orcid.org/0000-0002-8001-911X

\section{REFERENCES}

1 Merskey $\mathrm{H}$. Classification of chronic pain: descriptions of chronic pain syndromes and definitions of pain terms. Pain 1986;3:226.

2 Williams ACdeC, Craig KD. Updating the definition of pain. Pain 2016;157:2420-3.

3 Lavand'homme P. The progression from acute to chronic pain. Curr Opin Anaesthesiol 2011;24:545-50.

4 Treede R-D, Rief W, Barke A, et al. A classification of chronic pain for ICD-11. Pain 2015;156:1-7.

5 Bevan S, Quadrello T, Mcgee R, et al. Fit for work pain-European report 2009.

6 Barke A, Schiller J, Rief W, et al. The IASP classification of chronic pain for ICD-11. Pain 2018;160:88-94.

7 Breivik H, Collett B, Ventafridda V, et al. Survey of chronic pain in Europe: prevalence, impact on daily life, and treatment. Eur J Pain 2006;10:287-333.

8 Larsson C, Hansson EE, Sundquist K, et al. Chronic pain in older adults: prevalence, incidence, and risk factors. Scand J Rheumatol 2017;46:317-25.

9 Nahin RL. Estimates of pain prevalence and severity in adults: United States, 2012. J Pain 2015;16:769-80.

10 Häuser W, Wolfe F, Henningsen $P$, et al. Untying chronic pain: prevalence and societal burden of chronic pain stages in the general population - a cross-sectional survey. BMC Public Health 2014;14:1-8.

11 Miró J, Paredes S, Rull M, et al. Pain in older adults: a prevalence study in the Mediterranean region of Catalonia. Eur J Pain 2007;11.

12 Vincent GE, Velkoff VA. The next four decades the older population in the United States : 2010 to 2050. Vol. 2011. U.S. Department of Commerce. Economics and Statistics Administration. U.S. Census Bureau, 2010.

13 Breivik H, Eisenberg E, O'Brien T. The individual and societal burden of chronic pain in Europe: the case for strategic prioritisation and action to improve knowledge and availability of appropriate care. BMC Public Health 2013;13.

14 Frießem CH, Willweber-Strumpf A, Zenz MW. Chronic pain in primary care. German figures from 1991 and 2006. BMC Public Health 2009;9:299.

15 Vos T, Barber RM, Bell B, et al. Global, regional, and national incidence, prevalence, and years lived with disability for 301 acute and chronic diseases and injuries in 188 countries, 1990-2013: a systematic analysis for the global burden of disease study 2013. Lancet 2015;386:743-800.

16 Blyth FM, Van Der Windt DA, Croft PR. Chronic disabling pain. Am J Prev Med 2015;49:98-101.

17 Gatchel RJ, McGeary DD, McGeary CA, et al. Interdisciplinary chronic pain management: past, present, and future. Am Psychol 2014:69:119-30.

18 Geneen LJ, Moore RA, Clarke C, et al. Physical activity and exercise for chronic pain in adults: an overview of Cochrane reviews. Cochrane Database Syst Rev 2017;1.

19 Hughes LS, Clark J, Colclough JA, et al. Acceptance and commitment therapy (act) for chronic pain: a systematic review and meta-analyses. Clin J Pain 2017;33:552-68.

20 Dansie EJ, Turk DC. Assessment of patients with chronic pain. $\mathrm{Br} \mathrm{J}$ Anaesth 2013;111:19-25.

21 Suso-Ribera C, Castilla D, Zaragozá I, et al. Validity, reliability, feasibility, and usefulness of pain monitor: a multidimensional smartphone APP for daily monitoring of adults with heterogenous chronic pain. Clin J Pain 2018;34:1-8.

22 Jensen MP, McFarland CA. Increasing the reliability and validity of pain intensity measurement in chronic pain patients. Pain 1993;55:195-203.

23 Kikuchi H, Yoshiuchi K, Miyasaka N, et al. Reliability of recalled self-report on headache intensity: investigation using ecologica momentary assessment technique. Cephalalgia 2006;26:1335-43.

24 Kratz AL, Murphy SL, Braley TJ. Ecological Momentary assessment of pain, fatigue, depressive, and cognitive symptoms reveals significant daily variability in multiple sclerosis. Arch Phys Med Rehabil 2017;98:2142-50.
25 García-Palacios A, Herrero R, Belmonte MA, et al. Ecological momentary assessment for chronic pain in fibromyalgia using a smartphone: a randomized crossover study. EJP 2014;18:862-72.

26 Smyth JM, Smyth JM, Stone AA. Ecological Momentary assessment research in behavioral medicine. J Happiness Stud 2003;4:35-52.

27 Shiffman S, Stone AA, Hufford MR. Ecological Momentary assessment. Annu Rev Clin Psychol 2008;4:1-32.

28 Lin W-C, Burke L, Schlenk EA, et al. Use of an ecological Momentary assessment application to assess the effects of auricular point Acupressure for chronic low back pain. CIN: Computers, Informatics, Nursing 2019;37:276-82.

29 Suso-Ribera C, Mesas Ángela, Medel J, et al. Improving pain treatment with a smartphone APP: study protocol for a randomized controlled trial. Trials 2018;19:145.

30 May M, Junghaenel DU, Ono M, et al. Ecological Momentary assessment methodology in chronic pain research: a systematic review. J Pain 2018;19:699-716.

31 Moore J. The benefits of mobile apps for patients and providers. $\mathrm{Br} \mathrm{J}$ Healthc Manag 2012;18:465-7.

32 Reynoldson C, Stones C, Allsop M, et al. Assessing the quality and usability of smartphone Apps for pain self-management. Pain Med 2014;15:898-909.

33 Rosser BA, Eccleston C. Smartphone applications for pain management. J Telemed Telecare 2011;17:308-12.

34 Nieto R, Raichle KA, Jensen MP, et al. Changes in pain-related beliefs, coping, and catastrophizing predict changes in pain intensity, pain interference, and psychological functioning in individuals with myotonic muscular dystrophy and facioscapulohumeral dystrophy. Clin J Pain 2012;28:47-54.

35 Faul F, Erdfelder E, Lang A-G, et al. G*Power 3: a flexible statistical power analysis program for the social, behavioral, and biomedical sciences. Behav Res Methods 2007;39:175-91.

36 Kristjánsdóttir Ólöf Birna, Fors EA, Eide E, et al. A smartphonebased intervention with diaries and therapist-feedback to reduce catastrophizing and increase functioning in women with chronic widespread pain: randomized controlled trial. J Med Internet Res 2013;15:e5.

37 Macea DD, Gajos K, Daglia Calil YA, et al. The efficacy of web-based cognitive behavioral interventions for chronic pain: a systematic review and meta-analysis. J Pain 2010;11:917-29.

38 Urbaniak GC, Plous S. Research randomizer (version 4.0)[computer software]. Social Psychology Network 2013.

39 Kaiser U, Kopkow C, Deckert S, et al. Developing a core outcome domain set to assessing effectiveness of interdisciplinary multimodal pain therapy: the VAPAIN consensus statement on core outcome domains. Pain 2018;159:673-83.

40 Dworkin RH, Turk DC, Farrar JT, et al. Core outcome measures for chronic pain clinical trials: IMMPACT recommendations. Pain 2005;113:9-19.

41 Attal N, Cruccu G, Baron R, et al. EFNS guidelines on the pharmacological treatment of neuropathic pain: 2010 revision. Eur $J$ Neurol 2010;17:1113-88.

42 Varrassi G, Müller-Schwefe G, Pergolizzi J, et al. Pharmacological treatment of chronic pain - the need for change. Curr Med Res Opin 2010;26:1231-45.

43 Cleeland CS, Ryan KM. Pain assessment: global use of the brief pain inventory. Ann Acad Med Singapore 1994;23:129-38.

44 Sullivan MJL, Bishop SR, Pivik J. The pain Catastrophizing scale: development and validation. Psychol Assess 1995;7:524-32.

45 Zigmond AS, Snaith RP. The hospital anxiety and depression scale. Acta Psychiatr Scand 1983;67:361-70.

46 Gorin AA, Stone AA. Recall biases and cognitive errors in retrospective self-reports: A call for momentary assessments. In: Baum A, Revenson T, Singer J, eds. Handbook of health psycholog. Mahwah, NJ: Lawrence Erlbaum, 2001: 405-13.

47 Schwarz N. Retrospective and Concurrent Self-Reports: The Rationale for Real-Time Data Capture. In: Stone A, Shiffman SS, Atienza A, et al, eds. The science of real-time data capture: Self-reports in health research. New York: Oxford University Pres, 2007: 11-26.

48 Alexander J, Joshi G. Smartphone applications for chronic pain management : a critical appraisal. J Pain Res 2016;9:731-4.

49 Kantar World Panel. Smartphone OS sales market share, 2015. Available: http://www.kantarworldpanel.com/global/smartphone-osmarket-share [Accessed Retrieved 5 May 2019]. 